\title{
Hepatic veno-occlusive disease due to tacrolimus in a single-lung transplant patient
}

\author{
S. Shah*, M. Budev*, H. Blazey*, K. Fairbanks* and A. Mehta*
}

ABSTRACT: Hepatic veno-occlusive disease is defined as nonthrombotic fibrous obliterative endophlebitis of small centrilobular hepatic venules.

Clinically, patients present with elevated liver enzymes and a triad of jaundice, hepatomegaly and ascites. Although reported as a complication of other solid organ and stem cell transplantation, there have been no reported cases to date of veno-occlusive disease following lung transplantation.

The present authors report a case of veno-occlusive disease following single-lung transplantation in a patient on a triple-drug immunosuppressive regimen composed of tacrolimus, mycophenolate mofetil and prednisone.

The diagnosis was established by transjugular liver biopsy and by discontinuing tacrolimus; there was clinical regression of symptoms and serological return to baseline.

\section{KEYWORDS: Lung transplantation, tacrolimus, veno-occlusive disease}

$\mathbf{V}$ eno-occlusive disease (VOD), also known as Chiari's syndrome, was first described in the early 1920s in South Africa as a result of senecio poisoning and the ingestion of medicinal teas containing pyrrolizidine alkaloids $[1,2]$. The term hepatic veno-occlusive disease (HVOD) was coined 30 yrs later, to describe the nonthrombotic, fibrous obliterative endophlebitis of the small centrilobular hepatic venules. Recently, the term sinusoidal obstruction syndrome (SOS) has been proposed to replace HVOD, since SOS more accurately describes the toxic injury to the hepatic sinusoids. The clinical findings of hepatomegaly, ascites and jaundice compose the classical triad used to diagnose HVOD [3, 4]. VOD is now best recognised as a complication of high-dose immunosuppressive therapy in solid-organ transplantation.

\section{CASE REPORT}

A 60-yr-old female with a history of tobacco abuse underwent a cadaveric single left-lung transplant for severe chronic obstructive pulmonary disease. The patient had an uneventful postoperative recovery period and was discharged on an immunosuppressive drug regimen, which was composed of tacrolimus, mycophenolate mofetil, prednisone and dapsone. An elevated
Epstein-Barr virus titre was found during routine serological testing 2 months post-transplantation and oral valganciclovir therapy was initiated. Approximately 6 months after transplantation, she began to have symptoms of night sweats, early satiety and abdominal bloating with a 4.5$6.8-\mathrm{kg}$ weight loss. An aggressive malignancy work-up was pursued, which was unremarkable.

The patient reported development of low-grade fever, weight gain of $2.3 \mathrm{~kg}$ over her baseline and an increase in abdominal girth, 18 months after transplantation.

She had recently discontinued mycophenolate mofetil due to a low lymphocyte count and denied any addition of new medications. Physical examination revealed evidence of new ascites, and the tender, smooth, liver edge was palpable, $4-5 \mathrm{~cm}$ below the ribcage muscle, with no evidence of splenomegaly. Her cardiac examination demonstrated no evidence of jugular venous distention, distant heart sounds or a pericardial rub. Serological testing demonstrated a dramatic rise in her transaminases and evidence of new hyperbilirubinaemia. An abdomen/pelvis computed tomography (CT) scan demonstrated ascites with evidence of portal hypertension, dilated main portal vein and
AFFILIATIONS

Depts of *Pulmonary, Allergy and Critical Care, and

\#Gastroenterology and Hepatology, Cleveland Clinic Foundation, Cleveland, OH, USA.

\section{CORRESPONDENCE}

M. Budev

Dept of Pulmonary

Allergy and Critical Care

Cleveland Clinic Foundation

9500 Euclid Avenue

Cleveland

OH 44195

USA

Fax: 12164458160

E-mail: Budevm@ccf.org

Received:

April 212005

Accepted after revision:

October 032005 


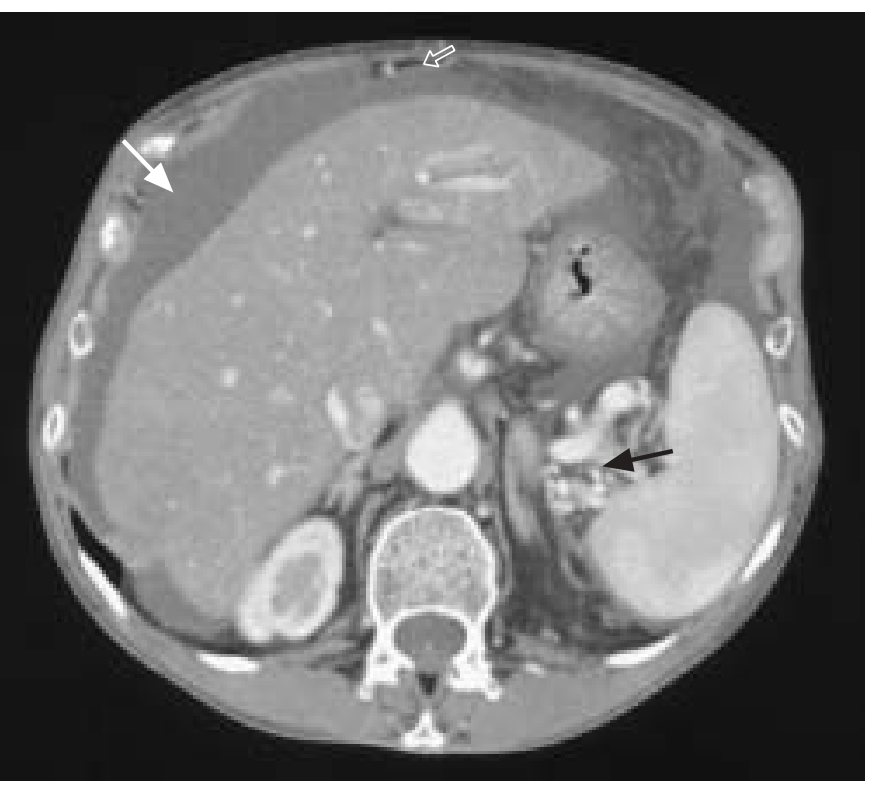

FIGURE 1. A computed tomography scan, demonstrating ascites (white arrow), revascularised para-umbilical vein (open arrow), dilated splenic vain and splenic hilar collaterals (black arrow). Diffuse hypo-attenuation of the liver is consistent with hepatic steatosis.

collaterals (fig. 1). The results of the liver function tests are shown in table 1.

An initial paracentesis attempt was aborted due to fluid loculation. A transjugular liver biopsy was obtained demonstrating centrilobular sinusoidal dilatation with haemorrhage and hepatocellular dropout. Trichrome staining demonstrated areas of centrilobular fibrosis and fibrous portal expansion without bridging fibrosis (fig. 2). The wedge hepatic pressure and portoatrial gradient were both recorded as $15 \mathrm{mmHg}$.

It was felt that the patient's symptoms, clinical presentation, elevated liver function tests and subsequent liver biopsy supported the diagnosis of HVOD, which was probably secondary to the tacrolimus. The patient was started on a treatment regime of spironolactone and furosemide to control her rapidly expanding ascites. Cyclosporin A was initiated to replace the tacrolimus. Within a 3-week period, there was resolution of her ascites, serum transaminases, as well as fever and malaise. Pentamidine was started for Pneumocystis carinii pneumonia prophylaxis and dapsone was discontinued.

\section{CASE DISCUSSION}

VOD has been described as a complication of high-dose chemotherapy, stem cell transplantation, radiation therapy and herbal toxicity [5]. Tacrolimus, a potent immunosuppressive agent, is now the most common calcinuric inhibitor used in thoracic organ transplantation in the USA. Although tacrolimus undergoes hepatic metabolism, only mild hepatotoxicity has been reported with its use. VOD of the liver after lung transplantation due to tacrolimus has never been reported in the literature.

Pre-existing liver damage, stem cell transplantation-related therapy and genetic polymorphism all appear to increase the risk of developing VOD. A specific polymorphism of the glutathione-S-transferase gene was found to be more frequent among patients who developed VOD. A better understanding of genetic polymorphism may be important in understanding the pathogenesis of VOD [6].

The clinical syndrome of VOD includes hepatomegaly, ascites and jaundice. HVOD is usually a difficult clinical diagnosis that may overlap with similar hepatic injury states, and it has a reported incidence of $10-60 \%$.

An abdominal CT and hepatic ultrasound showing the detection of reversed blood flow in the segmental branch of the portal vein using colour-flow Doppler is useful for the diagnosis of VOD. The gold standard of pathological diagnosis of VOD is the transjugular liver biopsy. A measurement of the wedged hepatic venous pressure gradient of $>10 \mathrm{mmHg}$ taken at the time of transjugular biopsy had a $91 \%$ specificity, $52 \%$ sensitivity and $86 \%$ positive predictive value [4].

Liver biopsies demonstrate dilation of the sinusoids and hepatic necrosis with sclerosis of the venular walls and intense collagen deposition in the sinusoids. This results in the obliteration of the venules, causing extensive hepatocellular necrosis and fibrous replacement of the hepatic tissue.

The prognosis of VOD depends on the extent of hepatic injury and dysfunction. Mild-to-moderate disease is characterised by liver dysfunction requiring diuretics and analgesics with eventual resolution of signs. Most patients fall into the mildto-moderate category; a significant fraction will develop severe VOD with a poor outcome requiring liver transplantation [5].

Little information exists in the way of treatment for VOD, but prevention is the key. Most established practice in the prevention of VOD is the use of the lowest possible drug

TABLE 1 Results of the liver function tests

\section{Date (DD/MM/YY)}

\begin{tabular}{|c|c|c|c|c|c|c|}
\hline & $28 / 12 / 04$ & $30 / 12 / 04$ & $21 / 01 / 05$ & $28 / 03 / 05$ & $25 / 04 / 05$ & $31 / 05 / 05$ \\
\hline Alkaline phosphatase $U \cdot L^{-1}$ & 449 & 381 & 398 & 518 & 436 & 323 \\
\hline ALT U. $L^{-1}$ & 59 & 41 & 58 & 78 & 68 & \\
\hline Bilirubin $\mathrm{mg} \cdot \mathrm{dL}^{-1}$ & 2.1 & 1.9 & 2.5 & 3.3 & 3.4 & 2.5 \\
\hline
\end{tabular}

AST: aspartate aminotransferase; ALT: alanine transaminase. 


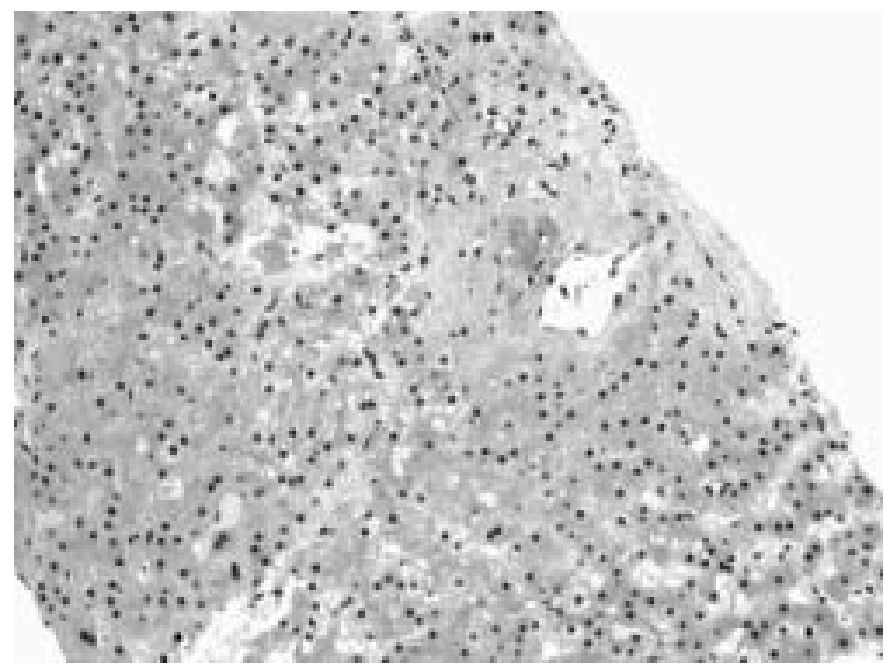

FIGURE 2. The perivenular (zone 3) region of the liver, showing centrilobar hepatocyte atrophy, dropout and haemorrhage. These changes are suggestive of venous outflow obstruction but can be seen in other instances, such as ischaemia or drug reactions.

dosing to minimise hepatic injury. Prostaglandin E1, a potent vasodilator, may play a part in decreasing the incidence and severity of VOD.

Managing patients consists of supportive care and avoidance of further hepatocellular damage or toxins, fluid restriction, discontinuing the immunosuppressive agent, analgesics, diuretics, paracentesis, correcting coagulopathy and controlling infection. Procedures, such as transjugular intrahepatic portosystemic shunts for cirrhosis and bleeding oesophageal varices, have been evaluated for the treatment of VOD in stem cell transplants [7]. Recently, recombinant tissue plasminogen activator was shown to be a possible therapy to reverse VOD.
In conclusion, veno-occlusive disease is clinically characterised as a triad of hepatomegaly, ascites and jaundice. This is the first case of veno-occlusive disease seen after a lung transplantation following immunosuppressive therapy. Further studies and research are needed to understand the pathophysiology of veno-occlusive disease. Promising research in hepatic cytoprotection and the mechanisms of hepatic fibrosis may suggest future therapeutic strategies.

\section{REFERENCES}

1 Selzer G, Parker R. Senecio poisoning exhibiting as Chiari's syndrome: a report on twelve cases. Am J Pathol 1951; 27: 885-887.

2 DeLeve L, Shulman HM, McDonald GB. Toxic injury to hepatic sinusoids: sinusoidal obstruction syndrome (venoocclusive disease). Semin Liver Dis 2002; 22: 27-42.

3 Shulman HM, Gooley T, Dudley MD, et al. Utility of transvenous liver biopsies and wedged hepatic venous pressure measurements in sixty marrow transplant recipients. Transplantation 1995; 59: 1015-1022.

4 Martha W, Vincent $\mathrm{HO}$, Parisa M, et al. Hepatic venoocclusive disease: pathogenesis, diagnosis and treatment. Curr Opin Hematol 2003; 10: 451-462.

5 Wendell T, David Z, George G, et al. Role of macrophages and stellate cells in the pathogenesis of veno-occlusive disease: an electron microscopic case study. Exp Mol Pathol 2003; 75: 201-209.

6 Beutler E, Gelbart T, Kondo T, Matsunaga AT. The molecular basis of a case of gamma-glutamylcysteine synthetase deficiency. Blood 1999; 94: 2890-2894.

7 Fried MW, Connaghan DG, Sharma S, et al. Transjugular intrahepatic portosystemic shunt for the management of severe venoocclusive disease following bone marrow transplantation. Hepatology 1996; 24: 588-591. 